\title{
Electric polarizability of the deuteron and the nucleon-nucleon interaction
}

\author{
M. H. Lopes, J. A. Tostevin, and R. C. Johnson \\ Department of Physics, University of Surrey, Guildford, Surrey, England
}

(Received 19 January 1983)

\begin{abstract}
We find that a recent experimental determination of the electric polarizability of the deuteron does not yield any new information on the nucleon-nucleon interaction. Theoretical values for the central and tensor components of the polarizability are presented.
\end{abstract}

[ NUCLEAR STRUCTURE d; calculated electric polarizability. ]

\section{INTRODUCTION}

There exist, in the literature, a large number of independent calculations of the deuteron electric polarizability which utilize different model n-p interactions, $V_{\mathrm{np}}\left({ }^{3} S_{1}\right.$ ${ }^{3} D_{1}$ ), to bind the deuteron. ${ }^{1}$ Recently, the first empirical determination of the polarizability has also been reported. ${ }^{2}$ It is timely, therefore, that the extent to which theoretically calculated values are model dependent, and hence that an accurate experimental determination could discriminate between available model interactions, be investigated.

\section{THE DEUTERON STATIC POLARIZABILITY}

The change, $\Delta \epsilon$, in the deuteron binding energy, $\epsilon_{\mathrm{d}}$, due to the dipole component $C(\vec{r}, \vec{R})$ of the deuteron-target (of

$$
\Delta \epsilon(\overrightarrow{\mathbf{R}})=\sum_{J} \int d K K^{2}\left\langle{ }^{3} S_{1}+{ }^{3} D_{1}|C|{ }^{3} L_{J}(K)\right\rangle\left\langle{ }^{3} L_{J}(K)\right|
$$

where the continuum state, denoted ${ }^{3} L_{J}(K)$, has energy

$$
E_{K}=\hbar^{2} K^{2} / 2 \mu,
$$

and $\mu$ is the reduced mass of the n-p system. In general, $\Delta \epsilon$ comprises both central and rank-two tensor (of the $T_{R}$ type) terms, ${ }^{3}$ and for large $R$ takes the form

$$
\Delta \epsilon(\overrightarrow{\mathbf{R}})=-(Z e)^{2}\left(\alpha+3 \tau T_{R}\right) /\left(2 R^{4}\right) .
$$

It is the strength of the central term, $\alpha$, usually referred to as the static polarizability, which has been determined empirically by Rodning et al. ${ }^{2}$ through a study of departures from Rutherford scattering of low energy deuterons. The deduced value, $\alpha=0.70 \pm 0.05 \mathrm{fm}^{3}$, is larger than has been obtained theoretically to date. The need for an accurate estimate for $\tau$ derives from recent studies of low energy (d,p) stripping reactions. Here, the calculated tensor analyzing powers, $T_{2 q}$, are particularly sensitive to the deuteron $D$-state component, ${ }^{4}$ and also to the presence of tensor terms in the deuteron-target interaction. ${ }^{5}$ Thus, in order to obtain accurate $D$-state information (the asymptotic $D$ - to $S$-state ratio, $\eta$ ) from the measured $T_{2 q}$, those tensor force effects associated with $\tau$ must be reliably subtracted. ${ }^{6}$

Both the central and tensor components, $\alpha, \tau$, of the electric polarizability are a sum of terms

$$
a=a_{S S}+a_{S D}+a_{D D}, \quad(a=\alpha, \tau)
$$

charge $Z$ ) polarizing Coulomb interaction

$$
P(\overrightarrow{\mathrm{r}}, \overrightarrow{\mathbf{R}})=Z e^{2} /(1 /|\overrightarrow{\mathbf{R}}+\overrightarrow{\mathrm{r}} / 2|-1 / R)
$$

is given, in second order perturbation theory, by the expression

$$
\Delta \epsilon(\overrightarrow{\mathrm{R}})=\left\langle\phi_{\mathrm{d}}\left|C(\overrightarrow{\mathrm{r}}, \overrightarrow{\mathrm{R}}) Q G_{\mathrm{np}} C(\overrightarrow{\mathrm{r}}, \overrightarrow{\mathrm{R}})\right| \phi_{\mathrm{d}}\right\rangle .
$$

Here, $\overrightarrow{\mathbf{r}}(\overrightarrow{\mathbf{R}})$ is the relative (center of mass) coordinate of the n-p pair, $Q$ projects off the deuteron ground state, $\phi_{\mathrm{d}}$, and

$$
G_{\mathrm{np}}=\left(-\epsilon_{\mathrm{d}}-H_{\mathrm{np}}\right)^{-1}
$$

propagates the nucleons in the intermediate state. Showing the angular momentum coupling explicitly, Eq. (2) can be written schematically

$\left.|C|{ }^{3} S_{1}+{ }^{3} D_{1}\right) /\left(-\epsilon_{\mathrm{d}}-E_{K}\right)$

linear and quadratic in the deuteron $S$ and $D$ states. These terms shall be evaluated separately, in the following, to determine the relative importance of the $D$-state component of the deuteron.

\section{TREATMENT OF THE n-p CONTINUUM}

The weakly bound nature of the deuteron, and the long range of the dipole coupling term, means that low energy breakup completely dominates the integral over intermediate states in Eq. (3). Consequently, it is usually assumed that plane wave states will give a good representation of that part of the continuum relevant to the determination of Eq. (3). We shall investigate the accuracy of this approximation. More generally, the dominant intermediate breakup states in Eq. (3) are the ${ }^{3} P_{J}$ configurations, which arise from direct coupling to both the $S$ - and $D$-state components of the deuteron wave function. The present work shows ${ }^{3}$ that, with intermediate $P$ waves, the integrand peaks for $E_{K}<2 \mathrm{MeV}$. Inspection of the experimental $J=2$ mixing parameter ${ }^{7}$ shows that effects due to ${ }^{3} P_{2}-{ }^{3} F_{2}$ coupling can safely be neglected in this energy range. The only remaining contribution to Eq. (3), other than that from $P$ states, is quadratic in the deuteron $D$ state and arises for ${ }^{3} L_{J}={ }^{3} F_{2}$. This term has been calculated assuming free particle states for the low energy relative $F$ waves and was found to be completely negligible. In the follow- 
ing analysis, therefore, only pure ${ }^{3} P_{J}$ configurations need be considered.

The $a_{L L^{\prime}}\left(a=\alpha, \tau: L, L^{\prime}=S, D\right)$ are sums of bilinear combinations, $I(J: L)^{*} I\left(J: L^{\prime}\right)$, of integrals of the form

$$
I(J: L)=\int d r r^{3} f_{J}(K, r) u_{L}(r),
$$

where $f_{J}\left(u_{L}\right)$ is the radial wave function of the $P$-wave continuum (deuteron). The presence of the $r^{3}$ factor, together with the resulting dominance of the low energy breakup components, strongly suggests that the long range parts of the deuteron ground state wave function

$$
\begin{aligned}
& u_{0}(r)=A_{S} e^{-\gamma r} / r, \\
& u_{2}(r)=\eta A_{S}\left[1+3 /(\gamma r)+3 /(\gamma r)^{2}\right] e^{-\gamma r} / r,
\end{aligned}
$$

will be of particular importance in determining the $I(J: L)$, and hence the calculated polarizability. We therefore investigate the extent to which $\alpha$ and $\tau$ are in fact determined by $A_{S}$ and $\eta$.

\section{THE DEUTERON GROUND STATE WAVE FUNCTION}

As stated earlier, Eq. (3) is conventionally calculated by replacing the intermediate states by plane waves. ${ }^{1}$ In this limit, $\alpha_{S D}$ and $\tau_{S S}$ vanish identically, which is not the case when a realistic $J$-dependent $P$-wave n-p interaction, $V_{\mathrm{np}}(P)$, is used. Our intention here is to assess the sensitivity of the calculated $\alpha$ and $\tau$ to both the interaction $V_{\mathrm{np}}\left({ }^{3} S_{1}-{ }^{3} D_{1}\right)$, which binds the deuteron ground state, and the interaction $V_{\mathrm{np}}(P)$ which generates the intermediate $P$ waves. To investigate the former we have performed calculations for a range of available model deuteron wave functions $^{8,9}$ corresponding to different $A_{S}$ and $\eta$. To clarify the latter point, we have taken for $V_{\mathrm{np}}(P)$ : (i) the Mongan (case I) separable interaction, ${ }^{7}$ and (ii) the Reid local ${ }^{3} P_{J}$ interactions. ${ }^{8}$ In the case of the Mongan interaction, the continuum functions $f_{J}(K, r)$ can be expressed in analytic form, whereas, for the Reid interaction they are obtained by direct numerical integration of the Schrödinger equation. Calculations have also been performed in the plane wave limit, $V_{\text {np }}(P)=0$. The two model $\boldsymbol{P}$-wave interactions used are phase equivalent, in that they both reproduce the experimental ${ }^{3} P_{J}$ phase shifts, but possess very different off-shell behavior. They thus permit a check to be made of whether the results of the calculation are sensitive to more than simply the $P$ wave phase shift, and hence whether the polarizability values obtained are model independent with regard to $V_{\text {np }}(P)$. Unless otherwise stated, all calculations presented use the Mongan interaction introduced above.

\section{RESULTS AND DISCUSSION}

Calculations for $\alpha$ show $\alpha_{S S}$ to be the completely dominant term. $\alpha_{S D}$ and $\alpha_{D D}$ contribute less than $0.5 \%$ of $\alpha$ and we do not consider these individually any further. For $\tau$, however, no term is negligible; $\tau_{S S}$ and $\tau_{D D}$ contribute approximately $14 \%$ and $5 \%$ of $\tau$, respectively. Clearly plane wave calculations for $\tau_{L L^{\prime}}$ are therefore in error. In Fig. 1 the calculated $\alpha$ are plotted against $A_{S}^{2}$ for

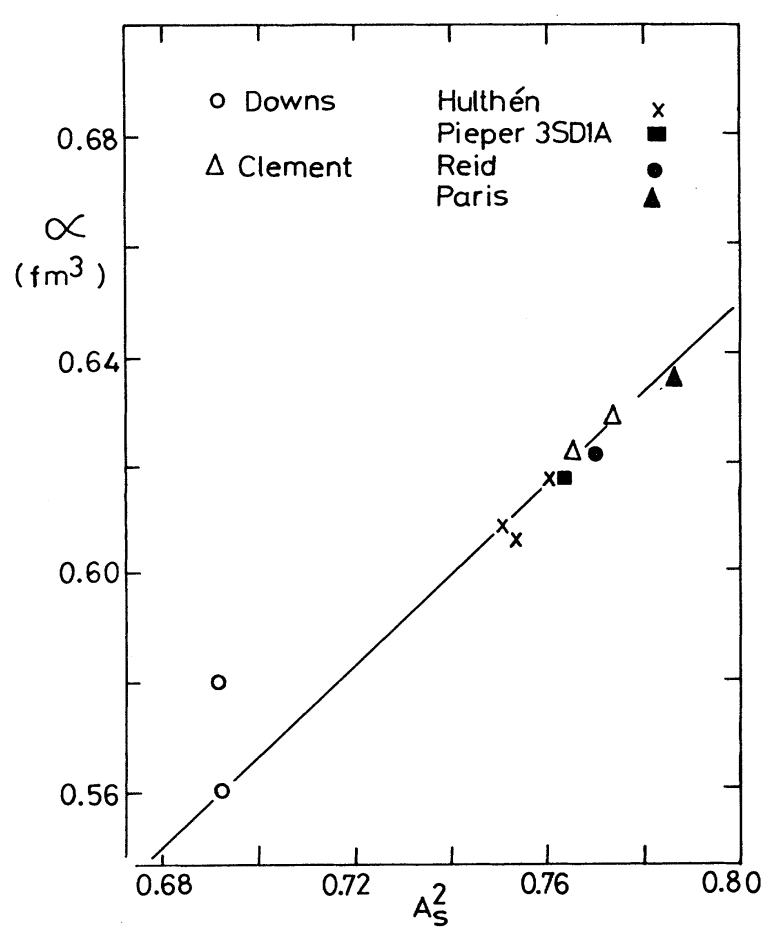

FIG. 1. Calculated deuteron polarizabilities, $\alpha$, for a number of model deuteron wave functions plotted versus the square of the asymptotic $S$-state normalization, $A_{S}^{2}$. The solid line is the theoretical prediction of Levinger (Ref. 10).

the model deuteron wave functions indicated.,9 Calculations from the literature ${ }^{1}$ are represented by open symbols and those from the present work by solid symbols. The calculated values of $\alpha$ are insensitive to whether the Mongan, Reid $(\Delta \alpha=0.1 \%)$, or plane wave $(\Delta \alpha=0.3 \%)$ intermediate state propagator is used. The $\alpha$ values obtained lie, with remarkable accuracy, upon a straight line of gradient $0.81 \mathrm{fm}^{4}$. The only value which does not conform to this empirical relation is the value $\alpha=0.58 \mathrm{fm}^{3}$ of Downs. ${ }^{1}$ The larger value obtained in this case is the result of Downs having used a strongly attractive effective $P$-wave interaction in the intermediate states, with a strength appropriate to the ${ }^{3} S_{1}-{ }^{3} D_{1}$ channel, and is therefore unphysical. As a consequence, the continuum function $f_{J}$, in Eq. (6) is enhanced at small n-p separations, increasing its overlap with the deuteron wave function, and hence the polarizability value obtained.

The origin of the value of the proportionality constant is best understood from the work of Levinger, ${ }^{10}$ which relates the deuteron polarizability to the deuteron inverse energy weighted photosum, $\sigma_{-2}$. Using the BetheLongmire effective range formula for the deuteron photoabsorption cross section, Levinger obtains the relation

$$
\alpha=e^{2} \hbar^{2} /\left(64 \mu \epsilon_{\mathrm{d}}^{2}\right) /\left[1-\gamma \rho\left(-\epsilon_{\mathrm{d}},-\epsilon_{\mathrm{d}}\right)\right] .
$$

However, in the shape-independent approximation, ${ }^{11} A_{S}$ is also determined to very high precision by the experimental effective range $\rho\left(-\epsilon_{\mathrm{d}},-\epsilon_{\mathrm{d}}\right)$, by the equation

$$
A_{S}^{2}=2 \gamma /\left[1-\gamma \rho\left(-\epsilon_{\mathrm{d}},-\epsilon_{\mathrm{d}}\right)\right],
$$


and therefore, a direct relationship between $\alpha$ and $A_{S}^{2}$ is obtained:

$$
\alpha=e^{2} /\left(64 \gamma^{3} \epsilon_{\mathrm{d}}\right) A_{S}^{2} .
$$

Alternatively, this same formula can been obtained (Clement, Ref. 1), using second order perturbation theory, if the asymptotic form of the deuteron wave function, Eq. (7a), is assumed to be valid for all $r$, and plane wave intermediate $\boldsymbol{P}$ states are used. Evaluation of the numerical constant, in Eq. (10), yields the value $0.81 \mathrm{fm}^{4}$, as obtained from the second order perturbation theory calculations which use realistic deuteron wave functions.

In Fig. 2, we consider the extent to which the $\tau_{L L^{\prime}}$ are determined by $A_{S}$ and $\eta$. For the model deuteron wave functions of Fig. 1 we plot, in addition to $\alpha / A_{S}^{2}$, the quantities $\tau_{S S} / A_{S}^{2}, \tau_{S D} / \eta A_{S}^{2}$, and $\tau_{D D} / \eta^{2} A_{S}^{2}$. The agreement between different models is extremely good. When the Reid $V_{n p}(P)$ is used in the intermediate states, instead of that of Mongan, the calculated $\tau$ change by less than $1 \%$, and show that $\tau$ is model independent in regard to $V_{\mathrm{np}}(P)$, provided the interaction reproduces the experimental phase shifts. We note, however, from Fig. 2, that there remains some residual model dependence in the calculated $\alpha$ and $\tau$ to the particular deuteron wave function used, that is the nonasymptotic part of the wave function.

To further investigate this sensitivity to the short range behavior of the deuteron wave function, we have applied finite range unitary transformations to the radial function, $u_{L}(r)$, generated by the Reid soft-core interaction. ${ }^{8}$ That is, for $r<2 \mathrm{fm}$, where our theoretical knowledge is incomplete, the deuteron wave function can be modified in an arbitrary manner, to $\bar{u}_{L}(r)$, for a given $g(r)(=0$, when $r>2 \mathrm{fm}$ ), according to

$$
\bar{u}_{L}(r)=u_{L}(r)+C_{L} g(r) \int d s s^{2} g(s) u_{L}(s),
$$

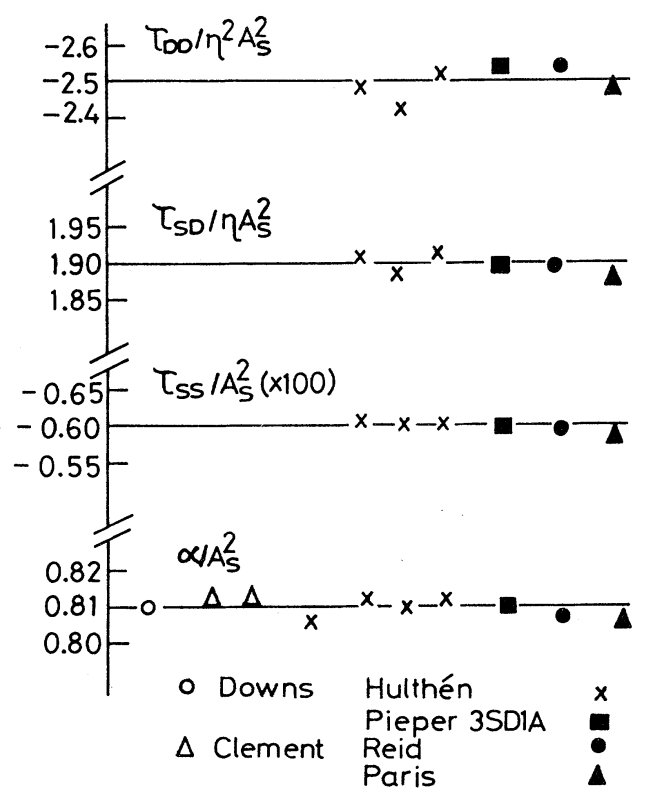

FIG. 2. Calculated values for $\alpha / A_{S}^{2}, \tau_{S S} / A_{S}^{2}, \tau_{S D} / \eta A_{S}^{2}$, and $\tau_{D D} / \eta^{2} A_{S}^{2}$ for a number of model deuteron wave functions. where $C_{L}$ must be chosen to preserve the norm. Beyond 2 fm, for given $A_{S}$ and $\eta$, the $u_{L}$ are essentially determined by the one pion exchange tail of $V_{\text {np }}$ (Ref. 11), are theoretically well understood, and thus remain fixed. We have taken, for $g(r)$, the transformation of Allen et al., ${ }^{12}$ and varied $\beta$, which governs the precise radial form of $g(r)$, in the range $-5-5$. The results of this analysis are shown in Fig. 3 as percentage changes to the calculated $\alpha$ and $\tau$ as a function of the transformation parameter $\beta$. It is evident that there is very little sensitivity in calculated polarizabilities to uncertainties in the precise form of the deuteron wave function at small n-p separations. Moreover, the $\alpha, \tau$ obtained for given $A_{S}, \eta$ cannot be increased by modification of the short range behavior of the $u_{L}(r)$.

The deuteron polarizability, as defined by Eqs. (1) and (3), is therefore theoretically and experimentally, through $A_{S}, \eta$, and the $P$-wave phase shifts, well determined. For a model deuteron wave function of given $A_{S}$ and $\eta$, then to a very good approximation (see Fig. 2)

$$
\begin{aligned}
& \alpha=0.81 A_{S}^{2} \mathrm{fm}^{3}, \\
& \tau=A_{S}^{2}\left(1.90 \eta-2.5 \eta^{2}-0.006\right) \mathrm{fm}^{3},
\end{aligned}
$$

for $A_{S}, \eta$ in the range $0.83 \mathrm{fm}^{-1 / 2}<A_{S}<0.89 \mathrm{fm}^{-1 / 2}$, $0.025<\eta<0.030$. This latter relation could be particularly useful, as it makes available the means by which a selfconsistent subtraction of the tensor force effect arising from $\tau$, Eq. (4), can be made in the analysis of subCoulomb (d,p) reactions, where $\eta$ is itself determined.

While there is, at present, consistency between empirical and theoretical estimates of the asymptotic $D$ - to $S$-state ratio $\eta=0.0271 \pm 0.0008$ (Refs. 6 and 11 ), there is much discussion as to the precise value of $A_{S}$. Ericson and Rosa-Clot $^{13}$ recommend a value $A_{S}=0.8846 \pm 0.0008$

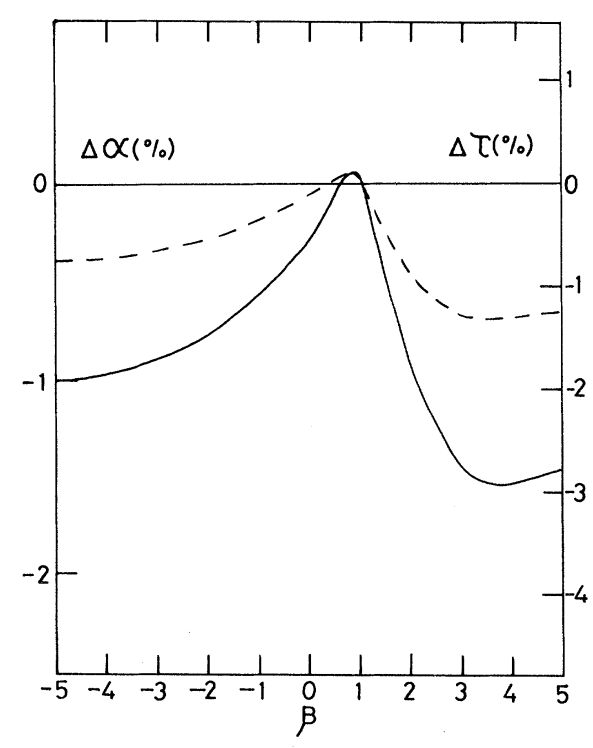

FIG. 3. Percentage changes in the polarizability strengths, $\alpha$ (solid curve) and $\tau$ (dashed curve), as a function of the unitary transformation parameter $\beta$, for the Reid soft-core wave function (Ref. 8). (The transformation parameter, $\alpha$, of Ref. 12 is taken as 1.5.) 
$\mathrm{fm}^{-1 / 2}$, whereas Kermode et al. ${ }^{14}$ obtain a value $0.8883 \pm 0.0044 \mathrm{fm}^{-1 / 2}$ with a considerably larger uncertainty. Although this latter value is known to be incorrect in detail, ${ }^{15}$ the associated error bar takes in the value of Ericson and Rosa-Clot, and the preliminary result of the reanalysis ${ }^{16}$ of the work of Ref. 14 . We take the value of Kermode et al., ${ }^{14}$ and its associated error, as being representative of the current state of knowledge of $\boldsymbol{A}_{S}$. With these values we obtain the results

$$
\begin{aligned}
& \alpha=0.639 \pm 0.006 \mathrm{fm}^{3}, \\
& \tau=0.0344 \pm 0.0013 \mathrm{fm}^{3},
\end{aligned}
$$

where the errors shown originate from $A_{S}$ and $\eta$ only.

In conclusion, calculations show that the scalar and tensor components of the deuteron polarizability are determined, to a precision far greater than that presently avail- able experimentally, by $A_{S}$ and $\eta$. A necessary element of such calculations is that one must use a $n-p$ interaction, $V_{\text {np }}(P)$, in the intermediate $P$-wave continuum which reproduces the experimental ${ }^{3} P_{J}$ phase shifts. The possibility of performing yet more precise deuteron elastic scattering experiments, of the type reported in Ref. 2, therefore provides, rather than a method of measuring the polarizability of the deuteron, the means by which one may investigate the validity of the polarizability concept, and understand, in detail, the reaction mechanism in low energy composite projectile scattering.

The financial support of the Instituto Nacional de Investigação Cientifica, Lisbon (for M.H.L.) and of the Science and Engineering Research Council (for J.A.T.) is gratefully acknowledged.
${ }^{1}$ N. F. Ramsey, B. J. Malenka, and V. E. Kruse, Phys. Rev. 91, 1162 (1953); B. W. Downs, ibid. 98, 194 (1955); C. F. Clement, ibid. 128, 2724 (1962); G. Baur, F. Rosel, and D. Trautmann, Nucl. Phys. A288, 113 (1977).

${ }^{2}$ N. L. Rodning, L. D. Knutson, W. G. Lynch, and M. B. Tsang, Phys. Rev. Lett. 49, 909 (1982).

${ }^{3}$ M. H. Lopes, J. A. Tostevin, and R. C. Johnson (unpublished).

${ }^{4}$ R. C. Johnson, Nucl. Phys. A99, 289 (1967).

${ }^{5}$ G. Delic and B. A. Robson, Nucl. Phys. A127, 234 (1969).

${ }^{6}$ R. P. Goddard, L. D. Knutson, and J. A. Tostevin, Phys. Lett. 118B, 241 (1982).

${ }^{7}$ T. R. Mongan, Phys. Rev. 178, 1597 (1969).

${ }^{8}$ R. V. Reid, Jr., Ann. Phys. (N.Y.) 50, 411 (1968).

${ }^{9}$ M. Lacombe, et al., Phys. Lett. 101B, 139 (1981); S. C. Pieper, Phys. Rev. C 9, 883 (1974); L. Hulthen and M. Sugawara, in
Handbuch der Physik, edited by S. Flugge (Springer, Berlin, 1957), Vol. 39, p. 1.

10J. S. Levinger, Phys. Rev. 107, 554 (1957).

${ }^{11}$ S. Klarsfeld, J. Martorell, and D. W. L. Sprung, Nucl. Phys. A352, 113 (1981).

${ }^{12}$ L. J. Allen, H. Fiedeldey, and N. J. McGurk, J. Phys. G 4, 353 (1978).

${ }^{13}$ T. E. O. Ericson and M. Rosa-Clot, CERN report No. TH3462-CERN, 1982.

${ }^{14}$ M. W. Kermode, A. McKerrell, J. P. McTavish, and L. J. Allen, Z. Phys. A 303, 167 (1981).

${ }^{15}$ D. W. L. Sprung, M. W. Kermode, and S. Klarsfeld, J. Phys. G $\underline{8}, 923$ (1982).

${ }^{16} \mathrm{M}$. W. Kermode (private communication). 